\title{
Operation of a load current multiplier on a nanosecond mega-ampere pulse forming line generator
}

\author{
A. S. Chuvatin, ${ }^{1}$ V. L. Kantsyrev, ${ }^{2}$ L. I. Rudakov, ${ }^{3}$ M. E. Cuneo, ${ }^{4}$ A. L. Astanovitskiy, ${ }^{2}$ R. Presura, ${ }^{2}$ A. S. Safronova, ${ }^{2}$ \\ W. Cline,${ }^{2}$ K. M. Williamson, ${ }^{2}$ I. Shrestha, ${ }^{2}$ G. C. Osborne, ${ }^{2}$ B. LeGalloudec, ${ }^{2}$ V. Nalajala, ${ }^{2}$ \\ T. D. Pointon, ${ }^{4}$ and K. A. Mikkelson ${ }^{4}$ \\ ${ }^{1}$ Laboratoire de Physique des Plasmas, Ecole Polytechnique, Palaiseau 91128, France \\ ${ }^{2}$ University of Nevada Reno, Reno, Nevada 89557, USA \\ ${ }^{3}$ Icarus Research, Inc., P.O. Box 30780, Bethesda, Maryland 20824, USA \\ ${ }^{4}$ Sandia National Laboratories, Albuquerque, New Mexico 87185, USA \\ (Received 8 November 2008; published 25 January 2010)
}

\begin{abstract}
We investigate the operation of a load current multiplier (LCM) on a pulse-forming-line nanosecond pulse-power generator. Potential benefits of using the LCM technique on such generators are studied analytically for a simplified case. A concrete LCM design on the Zebra accelerator (1.9 Ohm, 1 MA, $100 \mathrm{~ns}$ ) is described. This design is demonstrated experimentally with high-voltage power pulses having a rise time of dozens of nanoseconds. Higher currents and magnetic energies were observed in constantinductance solid-state loads when a better generator-to-load energy coupling was achieved. The load current on Zebra was increased from the nominal 0.8-0.9 MA up to about 1.6 MA. This result was obtained without modifying the generator energetics or architecture and it is in good agreement with the presented numerical simulations. Validation of the LCM technique at a nanosecond time scale is of importance for the high-energy-density physics research.
\end{abstract}

DOI: 10.1103/PhysRevSTAB.13.010401

PACS numbers: 84.70.+p, 84.60.Ve

Megagauss-range magnetic fields are widely applied in high-energy-density physics, plasma physics, solid-state physics, such as studies of material properties under high dynamic pressures, etc. [1]. Some applications including fast heating of highly radiating materials, inertial plasma confinement, or generation of strong shocks require also the shortest possible times of the magnetic energy release [2]. The most developed approach allowing high current pulses (mega-amperes) with short $\left(\sim 10^{-7} \mathrm{~s}\right)$ rise times is the pulsed-power technique based on pulse forming lines (PFL) [3]. In this technique, high magnetic fields can be obtained at small millimeter-scale diameters, usually in a solid-state or plasma load.

However, this approach requires more than tenfold concentration of the electromagnetic power in space from the large-size (dozens of centimeters) dielectric-insulated PFL output to a small, centimeter-scale vacuum volume representing the high magnetic energy density load. For a given value of the PFL voltage $V_{\text {oc }}$, the minimum physical volume necessary for electric connections to the load is constrained by high-voltage dielectric and vacuum insulation strengths. The higher the voltage $V_{\mathrm{oc}}$, the larger the connection volume and the higher its self-inductance $L_{0}$. In turn, the PFL impedance $\rho$ limits the maximum accessible load current amplitude $I_{0}$ to $V_{\text {oc }} / \rho$. The rise time $\tau \propto L_{0} / \rho$ of $I_{0}(t)$ is thus limited from below by the minimum $L_{0}$ volume which still ensures lossless energy delivery to the load [3]. A practical compromise between higher $I_{0}$ and smaller $\tau$ attainable at the present technology level of mega-ampere PFL's with $\tau \sim 100 \mathrm{~ns}$ results in $L_{0} \geq$ $10-20 \mathrm{nH}$.

At the same time, high magnetic energy densities are achievable in small load volumes which can have inductance $L_{d}$ of only units of nanohenries, so that $L_{d} \ll L_{0}$. At a given $I_{0}$ constrained by $\rho$, by $V_{\mathrm{oc}}$ and thus by $L_{0}$, see above, the driver-to-load energy transfer efficiency defined by the ratio $\eta=L_{d} /\left(L_{0}+L_{d}\right)$ can be also small. New generation pulse-power drivers with petawatt-range electromagnetic power would require an almost threefold increase of $V_{\mathrm{oc}}$ and $L_{0}$ [4], while the load volume and inductance $L_{d}$, or its variation $\Delta L_{d}$ remain unchanged for useful applications such as inertial confinement fusion [3]. This would lead to a further decrease of the efficiency $\eta$. It seems that a dramatic increase of $I_{0}$ can be accessible only through the increase of the generator output voltage $[3,4]$ that would require a larger and more expensive pulsepower system with larger $L_{0}$ and lower $\eta$.

The purpose of this paper is to demonstrate this is not always true and to show how the load magnetic energy can be enhanced without changing the generator architecture by decreasing $\rho$ and/or increasing the generator stored energy to allow $V_{\text {oc }}$ to be increased. We demonstrate a different approach to increasing the load current, the load current multiplier (LCM) [5]. This recently suggested method operates as an impedance transformer and increases the load magnetic energy. LCM's were previously tested with low-voltage, microsecond capacitor discharges [6] but have never been applied on PFL high-voltage, 
nanosecond-pulse generators. We now show an increase of the current by a factor of $1.88 \pm 0.38$, for $100 \mathrm{~ns}$ pulses with this technique on a nanosecond PFL generator Zebra [7-9] in the University of Nevada, Reno.

A PFL-based generator can be characterized by its wave impedance $\rho$ and by the open-circuit voltage $V_{\mathrm{oc}}$ at the dielectric-vacuum insulator [10]. The insulator and vacuum lines delivering the power to a load have some irreducible inductance $L_{0}$ which is limited from above by the minimum possible interelectrode gaps still allowing one to avoid insulator breakdown and electron current losses and plasma shorting of the vacuum gaps [3].

The Zebra generator [9] has an architecture typical for PFL-based pulse-power facilities and it is routinely used for production of strong magnetic fields [11] and in experimental $z$-pinch physics research [12]. Figure 1 shows the output section in standard generator operation with solidstate or plasma loads. We further consider only inductive loads having constant inductance $L_{d}$. Configurations of Fig. 1 then are described by the following equation [10]:

$$
\left(L_{0}+L_{\mathrm{dc}}+L_{d}\right) \frac{d I_{0}}{d t}+\rho I_{0}=V_{\mathrm{oc}}
$$

where $I_{0}$ is the output current and the inductances $L_{0}, L_{\mathrm{dc}}$, and $L_{d}$ are explained in the notation to Fig. 1.

Study a simplified case when the open-circuit voltage is constant, i.e., it instantaneously rises to the value $V_{\text {oc }}$ at $t=$ 0 and then remains unchanged. In other words, the voltage rise time $t_{0 \mathrm{~V}}=0$ in this approximation. Solution of Eq. (1) is

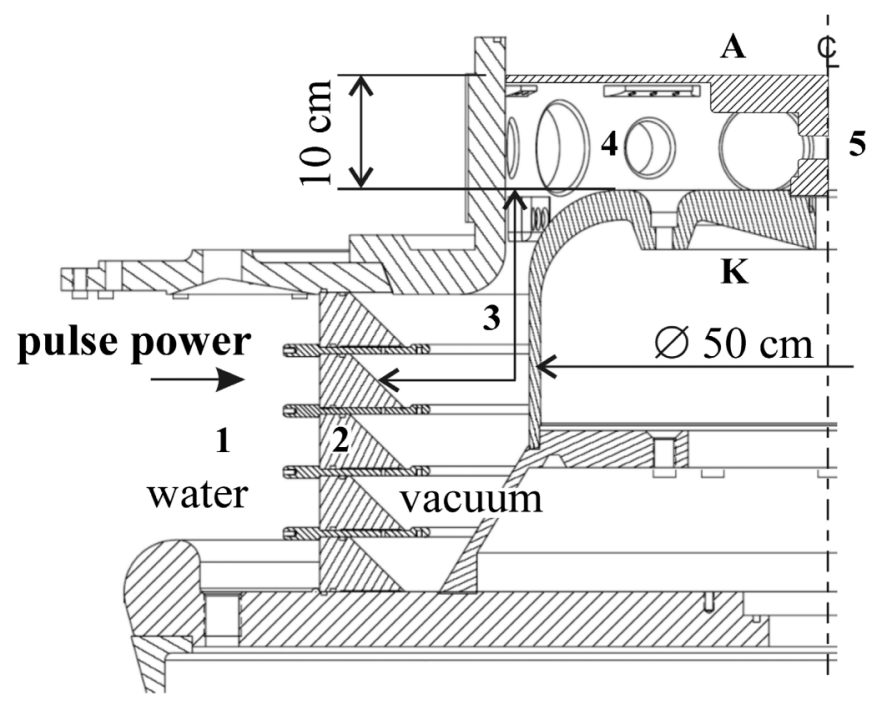

FIG. 1. Output section of the nanosecond pulse-power generator Zebra comprising (1) pulse forming line, (2) water-vacuum insulator stack, (3) vacuum pulse transmission line. Total inductance of the parts (2) and (3) is considered as the unchangeable generator inductance $L_{0}$. Diagnostic chamber (4) and the load (5) have the inductances $L_{\mathrm{dc}}$ and $L_{d}$, respectively. $A$-anode, $K$-cathode.

$$
I_{0}=\frac{V_{\mathrm{oc}}}{\rho}\left[1-\exp \left(-\frac{\rho}{L_{\mathrm{tot}}} t\right)\right]
$$

where $L_{\mathrm{tot}} \equiv L_{0}+L_{\mathrm{dc}}+L_{d}$. For $t \rightarrow \infty$, the generator and load current $I_{0}$ tends to the short-circuit value $V_{\mathrm{oc}} / \rho$. Let us define the characteristic load current rise time as $t_{0} \equiv 2 L_{\text {tot }} / \rho$ and let us introduce the characteristic load current amplitude $I_{0}{ }^{\max }$ as the current value at $t=t_{0}$, when $I_{0}{ }^{\max } \equiv I_{0}\left(t_{0}\right) \approx 0.86 V_{\text {oc }} / \rho:$

$$
I_{0}{ }^{\max } \equiv I_{0}\left(t_{0}\right)=\frac{V_{\mathrm{oc}}}{\rho}\left(1-e^{-2}\right)
$$

Compare this simple estimate to a typical experimental result in standard Zebra configuration of Fig. 1. Standard Zebra parameters are $\rho=1.9 \mathrm{Ohm}$ [7] for the generator impedance and $L_{0} \approx 26 \mathrm{nH}$ for the generator inductance corresponding to the volumes (2) and (3) in Fig. 1. $L_{\mathrm{dc}}$ and $L_{d}$ are the inductances of the diagnostic chamber (4) and load (5), respectively, and they change depending on the experiment. Figure 2 shows typical Zebra load current pulse measured in a calibration shot with $L_{\mathrm{dc}}+L_{d} \approx$ $40 \mathrm{nH}\left(L_{\text {tot }} \approx 66 \mathrm{nH}\right)$, as well as the generator opencircuit voltage $V_{\mathrm{oc}}(t)$. The waveform of $V_{\mathrm{oc}}$ was derived in a separate series of experiments (not discussed here) from the voltage measurements across the insulator stack, Fig. 1, together with $d I_{0} / d t$ measurements. As the experimental $V_{\mathrm{oc}}(t)$ had shot-to-shot variation in shape and in maximum amplitude, Fig. 2 presents the averaged value over eight consecutive shots with the maximum of $V_{\mathrm{oc}}=$ $1.98 \mathrm{MV}$ having a shot-to-shot variation of $\pm 13 \%$. The experimental voltage rise time is $t_{0 \mathrm{~V}} \sim 100 \mathrm{~ns}$ in Fig. 2.

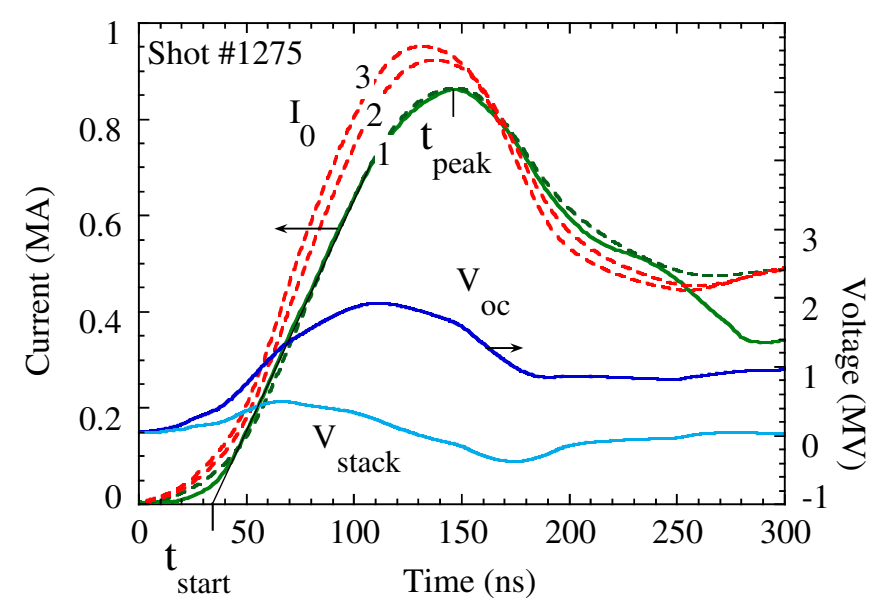

FIG. 2. (Color) Nominal short-pulse mode operation of the generator. (1) Experimental generator and load current $I_{0}$ for the shot with $L_{\mathrm{dc}}+L_{d} \approx 40 \mathrm{nH}$, solid line. Experimental averaged voltage across the stack, $V_{\text {stack }}$, and the unfolded opencircuit voltage $V_{\mathrm{oc}}$ (cannot be trusted for $t>200-250 \mathrm{~ns}$ ). (1) Numerical solution $I_{0}(t)$ of Eq. (1) for this shot using experimental $V_{\mathrm{oc}}(t)$, dashed line. Also shown for comparison numerical simulations with (2) $L_{\mathrm{dc}}+L_{d}=20 \mathrm{nH}$ and (3) $L_{\mathrm{dc}}+$ $L_{d}=10 \mathrm{nH}$. 
In this calibration shot, $I_{0}{ }^{\max }=0.86 \mathrm{MA}$ and the experimental current rise time defined here and below is $t_{d}{ }^{\exp }=t_{\text {peak }}-t_{\text {start }}\left(t_{\text {peak }}\right.$ corresponds to the maximum $I_{0}$ and $t_{\text {start }}$ is found from extrapolation of the linear pulse rise portion), so that experimentally $t_{d}^{\exp }=114 \mathrm{~ns}$. Substitution of the maximum experimental $V_{\mathrm{oc}}$ value in Eq. (3) yields $I_{0} \max \approx 0.9 \mathrm{MA}$, which is close to the experimentally measured $0.86 \mathrm{MA}$.

At the same time, the time constant $t_{0}$ defined above, $t_{0} \approx 69 \mathrm{~ns}$ appears to be substantially smaller than $t_{d}$ exp . This is because Eq. (2) was obtained, and $t_{0}$ was defined for an ideal steplike voltage waveform with $t_{0 \mathrm{~V}}=0$, when the current rise time is constrained only by the output inductance $L_{\text {tot }}$ and generator impedance $\rho$ in Eq. (1). One could conventionally discriminate here two regimes of PFL generator operation onto an inductive load: (1) the "loadlimited" regime with $t_{0} \gg t_{0 \mathrm{~V}}$, when the load current rise time is mainly defined by $L_{\text {tot }}$, Eq. (2) and (2) the "generator-limited" regime with $t_{0} \mathrm{v} \gg t_{0}$ and with $t_{d} \exp \sim t_{0 \mathrm{~V}}$. The latter case is closer to Zebra operation in its standard configuration of Fig. 1 with $L_{\text {tot }} \approx 66 \mathrm{nH}$ and $t_{0} \approx 69 \mathrm{~ns}$, when $t_{0 \mathrm{~V}} \sim 100 \mathrm{~ns}$ and $t_{d}{ }^{\exp }=114 \mathrm{~ns} \approx$ $t_{0 \mathrm{~V}}$.

However, the output section of Fig. 1 will be modified following recommendations of Ref. [5] and the effective output inductance $L_{\text {tot }}$ seen by the generator will be increased when using the load current multiplier technique, see Eq. (6) in Ref. [5] and Eq. (8) below. The simplified analysis for an ideal generator is important in this case because it shows new constraints imposed by LCM. When the output inductance increases, $t_{0}$ increases accordingly and the generator current at a given time thus should decrease.

Before we proceed with revisions of the Zebra output geometry, let us see what the maximum current the generator could provide in the standard setup of Fig. 1. Experimental dependence $V_{\mathrm{oc}}(t)$ allows numerical solution of Eq. (2) for the calibration shot with $L_{\mathrm{dc}}+L_{d} \approx 40 \mathrm{nH}$. This numerical solution is shown in Fig. 2 (1) and it corresponds well to the experimental current waveform. Now, rearrangement of the diagnostic chamber and load electrodes (4) and (5) in Fig. 1 in order to decrease the corresponding inductance $L_{\mathrm{dc}}+L_{d}$ would help somewhat in increasing the current amplitude. However, as numerical results (2) and (3) show in Fig. 2, the generator performance is not considerably improved as the peak current rises only by $10 \%$ and in any case cannot exceed the theoretical limit of $V_{\mathrm{oc}} / \rho \sim 1 \mathrm{MA}$.

Suppose now a modification of the Zebra output section, Fig. 1, applying the LCM technique. The load $L_{d}$ is a small volume with high magnetic energy density. As described elsewhere [5], an LCM is capable to redistribute the energy between the coupling inductance $L_{0}$ and load inductance $L_{d}$ operating as a transformer, or impedance adapter, and increasing the fraction of the total energy

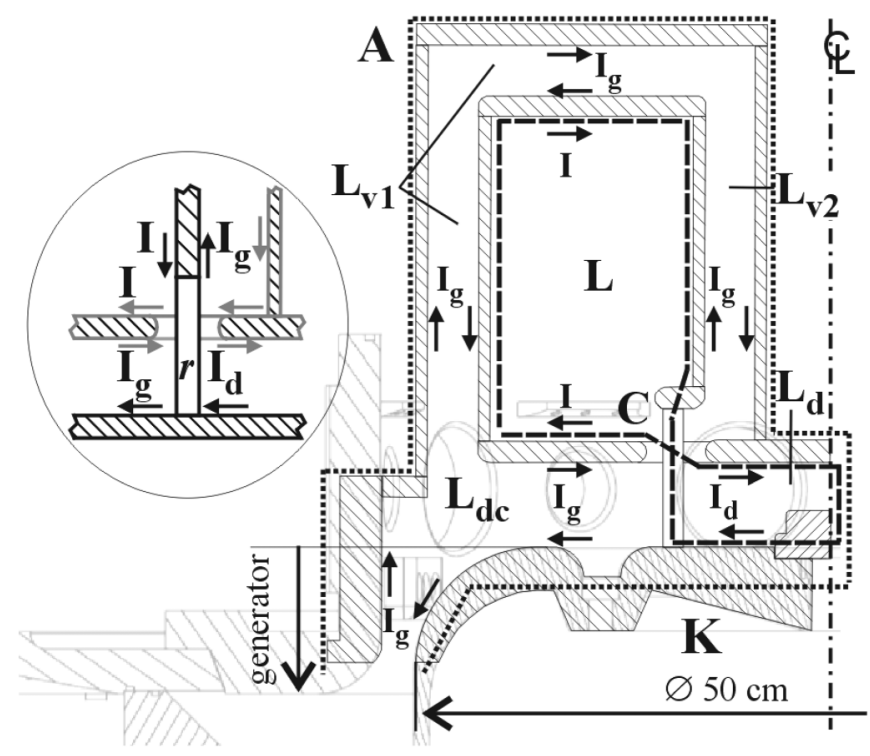

FIG. 3. A sketch of possible LCM implementation on Zebra in cylindrical geometry, cf. Fig. 1 . $A$-anode, $K$-cathode. $L_{\mathrm{dc}}$ is the diagnostic chamber inductance between the chamber wall and the LCM convolute posts, $L_{v}$ is the total bypass inductance of two coaxial and one disk lines, $L_{d}$ is the load inductance, and $L$ is inductance of the large LCM cavity. The arrows show directions of the currents in the system at the surfaces of electrodes. The current $I_{g}$ powered by PFL (not shown) passes through $L_{\mathrm{dc}}$ and then through the bypass $L_{v}$. Further two paths for $I_{g}$ to load are directly to $L_{d}$ and through the convolute $C$ and large volume $L$. At point $C$ the incoming generator current $I_{g}$ splits into surface currents $I, I_{d}$, and $I_{g}$ returning to the generator. Dotted and dashed contours are used for calculation of magnetic fluxes. The inset shows current splitting at the convolute $C$, where $r$ shows one convolute post.

available in the system that is coupled to the load at peak current.

Figure 3 suggests such a modification on Zebra and corresponds to the scheme of Fig. 1(b) from Ref. [5]. The LCM consists of two concentric toroids connected to the load $L_{d}$ through a post-hole convolute $C . L_{v}$ is the vacuum inductance between the toroids.

We still consider that the load inductance $L_{d}$ is constant and define it as that downstream of the convolute $C$, Fig. 3. We assume perfectly conducting electrodes and absence of current losses across the vacuum gaps everywhere in the system. The current $I_{g}$ comes from the generator and it flows on the inner surface of the external electrode of the vacuum chamber which is the anode $A$. The current flowing on the surface of the opposite electrode has the same value and opposite direction. $I_{g}$ finally returns to the generator on the surface of the central cathode $K$ (see also Fig. 1). The same current $I_{g}$ is on the surface of electrodes in the volumes $L_{v 1}$ and $L_{v 2}$, as well as in $L_{\mathrm{dc}}$. Current splitting occurs only at the convolute $C$ where four vacuum lines (volumes $L, L_{\mathrm{dc}}, L_{d}$, and $L_{v 2}$ ) are connected together, see the inset of Fig. 3. We chose clockwise direction of the 
currents $I$ and $I_{d}$ in the volumes $L$ and $L_{d}$ accordingly. Faraday's law for the external contour (dots) enclosing the generator and all the inductances shown in Fig. 3 implies

$$
\left(L_{0}+L_{\mathrm{dc}}+L_{v}\right) \frac{d I_{g}}{d t}+L \frac{d I}{d t}+L_{d} \frac{d I_{d}}{d t}+\rho I_{g}=V_{\mathrm{oc}}
$$

where $I_{g}$ is the generator current in the inductances $L_{0}, L_{\mathrm{dc}}$, and $L_{v}=L_{v 1}+L_{v 2}\left[I_{g}\right.$ is different from $I_{0}$ in Eq. (1) as the circuit is changed], $I_{d}$ is the load current in the inductance $L_{d}$, Fig. 3 , and $I$ is the current inside large cavity $L$ (magnetic flux extruder).

Then, use a contour including only the extruder inductance $L$ and the load $L_{d}$ (dashed contour in Fig. 3). We assume that the convolute inductance can be made small compared to that of the load. Choose counterclockwise path tracing of this contour so that the magnetic flux in $L$ will contribute as $-L I$ (direction of the current $I$ is opposite to the path-tracing direction), the flux in $L_{d}$ will contribute as $L_{d} I_{d}$ and the total flux will be equal to $-L I+$ $L_{d} I_{d}$. This magnetic flux is zero inside this contour before the power pulse because the currents are zero. This flux is further conserved because we assume absence of dissipation in perfectly conducting electrodes, so it remains zero when the generator current starts. Therefore, we have for this contour at any time

$$
L I-L_{d} I_{d}=0 .
$$

Current splitting at the convolute $C$ is illustrated by the inset of Fig. 3. Consider one convolute post $r$. The inflowing and outflowing currents for this post are shown by black arrows and they are $I+I_{d}$ and $I_{g}+I_{g}$ accordingly, so that the current continuity at the point $C$ requires

$$
I_{d}+I=2 I_{g}
$$

The same result can be obtained if we include more than one post or if we study inflowing and outflowing currents for the convolute holes (more precisely, for the spacing between holes) shown by gray arrows in the inset of Fig. 3.

Equations (4)-(6) thus describe the generator-LCMload circuit changed with LCM modification of the vacuum region. From Eqs. (5) and (6) the currents inside the extruder and into the load are thus

$$
I=\frac{2 L_{d}}{\left(L+L_{d}\right)} I_{g} \quad I_{d}=\frac{2 L}{\left(L+L_{d}\right)} I_{g} .
$$

Introducing the current multiplication coefficient $\kappa \equiv$ $I_{d} / I_{g}$, one can see that for $L \gg L_{d}$ we have $I \ll I_{g}$ and $\kappa \rightarrow 2$, i.e. if the extruder cavity inductance is much higher than that of the load, the extruder current becomes much smaller than that of the generator while the generator current can be doubled. The generator current differs from $I_{0}$ in Eq. (1) and it is now described by (4) or by the following, substituting Eq. (7) into Eq. (4):

$$
\begin{aligned}
& L_{\mathrm{tot}} * \frac{d I_{g}}{d t}+\rho I_{g}=V_{\mathrm{oc}} \\
& L_{\mathrm{tot}} * L_{0}+L_{\mathrm{dc}}+L_{v}+\frac{4 L L_{d}}{L+L_{d}} .
\end{aligned}
$$

Simplifying our analysis we consider once more $V_{\mathrm{oc}}(t)=$ const, i.e., the "load-limited" regime defined above. As discussed, studying this regime allows a comparison of LCM performance even with an ideal PFL generator. The solution of Eq. (8) for the generator current will be the same as (2) except $L_{\text {tot }}$ should be substituted by $L_{\mathrm{tot}}{ }^{*}$. Equation (7) allows one then to find the modified load current $I_{d}$ [cf. Eq. (2) defines the "old" current into load]:

$$
I_{d}=\frac{2 L}{\left(L+L_{d}\right)} \frac{V_{\mathrm{oc}}}{\rho}\left[1-\exp \left(-\frac{\rho}{L_{\mathrm{tot}}{ }^{*}} t\right)\right] .
$$

Similarly to Eq. (2), we define the new current rise time as $t_{d} \equiv 2 L_{\text {tot }}{ }^{*} / \rho$ and the new load current amplitude as $I_{d}{ }^{\max } \equiv I_{d}\left(t_{d}\right)$, so that at $t=t_{d}$ the current $I_{d}$ makes approximately $86 \%$ of its maximum possible value for $t \rightarrow$ $\infty$. Defined in this way, the $I_{d}{ }^{\max }$ value can reach 2 times the load current amplitude value $I_{0}{ }^{\max }$ of the standard nonLCM configuration (Fig. 1) if $L \gg L_{d}$, so that advantage of the LCM is evident:

$$
\begin{aligned}
& I_{g}{ }^{\max } \equiv I_{g}\left(t_{d}\right)=\frac{V_{\mathrm{oc}}}{\rho}\left(1-e^{-2}\right)=I_{0}{ }^{\max } \\
& I_{d}{ }^{\max } \equiv I_{d}\left(t_{d}\right)=\frac{2 L}{L+L_{d}} I_{g}{ }^{\max } \rightarrow 2 I_{0}{ }^{\max } \\
& i_{d}{ }^{\max } \equiv \frac{I_{d}{ }^{\max }}{I_{0}{ }^{\max }}=\frac{2 L}{L+L_{d}} \rightarrow 2 .
\end{aligned}
$$

However, as $L_{\mathrm{tot}}{ }^{*}>L_{\mathrm{tot}}$, the characteristic current rise time $t_{d}$ increases when the LCM is applied, i.e. $t_{d}>t_{0}$. In this case the load current maximum occurs later in time than in the no-LCM case and the $I_{d}$ value at $t=t_{0}$ is obviously lower than $I_{d}{ }^{\max }$. In order to investigate the efficiency of LCM operation in a more rigorous way, we normalize the load current of Eq. (9) to $I_{0}(t)$ from Eq. (2) and consider both $I_{d}(t)$ and $I_{0}(t)$ for $t=t_{0}, i_{d} \equiv$ $I_{d}\left(t_{0}\right) / I_{0}\left(t_{0}\right)$. The case $i_{d}>1$ would signify that the load current in Fig. 3 is higher even at the time where the noLCM current is maximum. In turn, dimensionless value $\tau \equiv t_{d} / t_{0}$ would characterize the degree of current rise time increase when the modification of Fig. 3 is considered. Expressions for the normalized load current $i_{d}$ and normalized current rise time $\tau$ are

$$
i_{d} \equiv \frac{I_{d}\left(t_{0}\right)}{I_{0}\left(t_{0}\right)}=\frac{2 L}{L+L_{d}} \frac{1-e^{-2 / \tau}}{1-e^{-2}} \quad \tau \equiv \frac{t_{d}}{t_{0}}=\frac{L_{\mathrm{tot}}{ }^{*}}{L_{\mathrm{tot}}} .
$$

For the sake of simplicity, suppose the value of $L_{\mathrm{dc}}$ to be the same both in Fig. 1 and in Fig. 3. Let us now neglect the bypass inductance value $L_{v}$, e.g. $L_{v} \ll L_{0}+L_{\mathrm{dc}}$ in 
Eq. (8). This condition is not automatically satisfied for all generators but it allows preliminary parametric study of a system with LCM. The normalized values of Eq. (11) can then be expressed as

$$
i_{d}=\frac{2 x}{d+x} \frac{1-e^{-2 / \tau}}{1-e^{-2}} \quad \tau=\frac{1+4 x d /(x+d)}{1+d},
$$

where $x \equiv L /\left(L_{0}+L_{\mathrm{dc}}\right)$ is the normalized extruder cavity inductance and $d \equiv L_{d} /\left(L_{0}+L_{\mathrm{dc}}\right)$ is the normalized load inductance.

The values of $i_{d}$ and $\tau$ from (12) are plotted in Fig. 4. This parametric analysis allows a preliminary estimate of the necessary LCM extruder volume $L$ for given $L_{0}+L_{\mathrm{dc}}$ and $L_{d}$. The LCM load current $I_{d}$ at $t=t_{0}$ can be made higher than the maximum current $I_{0}{ }^{\max }$ in standard generator configuration already at moderate extruder cavity inductance values. For example, for LCM with $x=2$ and for $d=0.25$, i.e., for $L_{d}=0.25\left(L_{0}+L_{\mathrm{dc}}\right)$, we have $I_{d} \approx$ $1.5 I_{0}{ }^{\max }$ at $t=t_{0}\left(i_{d} \approx 1.5\right.$ in Fig. 4). In turn, $i_{d}{ }^{\max }=$ $2 x /(x+d) \approx 1.78$ in Eq. (10) so that $I_{d} \approx 1.78 I_{0}{ }^{\max }$ at $t=t_{d}\left(t_{d} \approx 1.5 t_{0}\right.$ in Fig. 4). The region with $x<1$ for $d=$ 0.25 is not suitable even for an ideal PFL generator with $t_{0 \mathrm{~V}}=0$, Eq. (9), and an ideal LCM, $L_{v}=0$, because application of an LCM would lead to the load current rise time increase, $\tau>1$ with a moderate or no gain in the load current amplitude $i_{d}$. Similarly, the region with $x \gg 1$ should be more accurately studied from the viewpoint of a concrete engineering design because high $L$ lead to higher bypass inductance $L_{v}$, see Fig. 3, which may become non-negligible.

If we allow $t_{d}$ to be significantly greater than $t_{0}$, the LCM may potentially improve generator-to-load coupling and increase the load current above the no-LCM value even at higher load inductances than those presented in Fig. 4. For example, for $d=1\left(L_{d}=L_{0}+L_{\mathrm{dc}}\right)$, for $x \gg 1(L \gg$

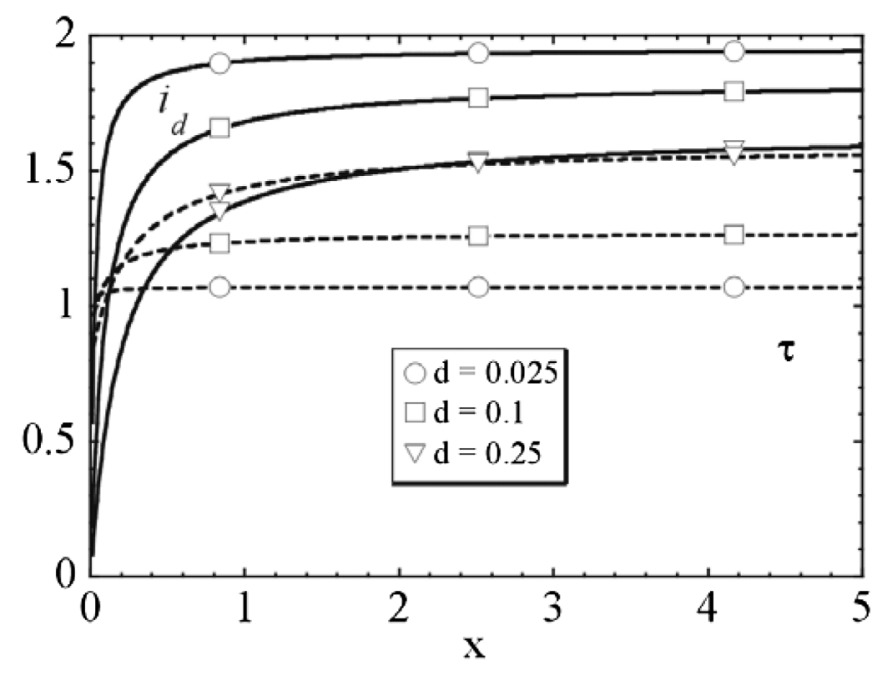

FIG. 4. Normalized load current $i_{d}$ (solid lines) and rise time $\tau$ (dashed lines) as functions of normalized LCM extruder inductance $x$ and for different normalized load inductances $d$, Eq. (12).
$\left.L_{0}+L_{\mathrm{dc}}\right)$, and when $L_{v}$ can be still neglected $\left(L_{v} \ll L_{0}+\right.$ $L_{\mathrm{dc}}$ ), Eqs. (10) and (12) yield $i_{d} \approx 1.27$ and $i_{d}{ }^{\max } \rightarrow 2$ for $t_{d} \rightarrow 2.5 t_{0}$.

Therefore, the simplified analysis above suggests that the LCM technique proposed in Ref. [5] is capable to improve characteristics of a PFL generator operating on inductive loads without changing the generator parameters $V_{\text {oc }}, \rho$, and $L_{0}$. For an ideal PFL generator able to deliver electrical power during sufficient time, with $V_{\mathrm{oc}}=$ constant, in the range of parameters discussed above the load current can be increased and have the same rise time $t_{0}$ as the no-LCM current. If the experiment allows $t_{d}>t_{0}$, the load current can be almost doubled.

Here we always considered inductive loads on PFL generators. Those loads correspond to a variety of solidstate and plasma loads in high-energy-density physics [113]. Figure 4 shows higher load current gains (higher $i_{d}$ at given $x$ ) for smaller normalized load inductances $d$. In an LCM configuration the load inductance $L_{d}$ is unambiguously defined as that downstream of the LCM convolute, Fig. 3. On the contrary, in a no-LCM case (standard pulsepower generator output, Fig. 1), the volume with constant inductance $L_{d}$ cannot be distinguished from the rest of the vacuum volume and this is the reason why we could use only the experiment-dependent sum value $L_{\mathrm{dc}}+L_{d}$, see Fig. 2.

In fact, LCM intentionally separates the small highenergy-density volume comprising physical processes under study from the rest of the vacuum volume in Fig. 2. In other words, the LCM technique implicitly assumes that (a) the load volume having constant inductance $L_{d}$ (e.g. for obtaining high pulse magnetic fields [11]) or variable inductance $\Delta L_{d}$ ( $z$ pinches, see, e.g., Ref. [12]) is a small volume with high magnetic energy density (the minimum size of this volume can be limited from below by some additional requirements, such as peculiarities of the physical process in interest, diagnostics access, etc.), and that (b) the LCM convolute is positioned as close as possible to $L_{d}$ (or $\Delta L_{d}$ ) volume. This may allow one to have $d \ll 1$ in the notations of Eq. (12) and thus $i_{d}>1$ in Fig. 4 or $i_{d}{ }^{\max } \rightarrow 2$ in Eq. (10).

Following this logic, we modify the vacuum section in Fig. 1 by adding additional hardware between the PFL output and the load as sketched in Fig. 3. The experimental hardware shown in Fig. 5 is designed to operate in vacuum and it comprises magnetically insulated transmission lines (MITL) $L_{v 1}$ and $L_{v 2}$, a large-volume magnetic flux extruder $L$ and a post-hole convolute $C$ (eight posts at the diameter of $18 \mathrm{~cm}$ ). The procedure of electrical description of this system presented above concludes that the generator current tends to pass 2 times through the load in an ideal case, when $L \gg L_{d}$, because in this case we have $I \rightarrow 0$ in Eq. (6) and thus $I_{d} \rightarrow 2 I_{g}$ in Eqs. (6) and (7).

In the experimental configuration of Fig. 5 the inductances in Eqs. (7) and (8) are: $L_{0} \approx 26 \mathrm{nH}$ (unchangeable), 


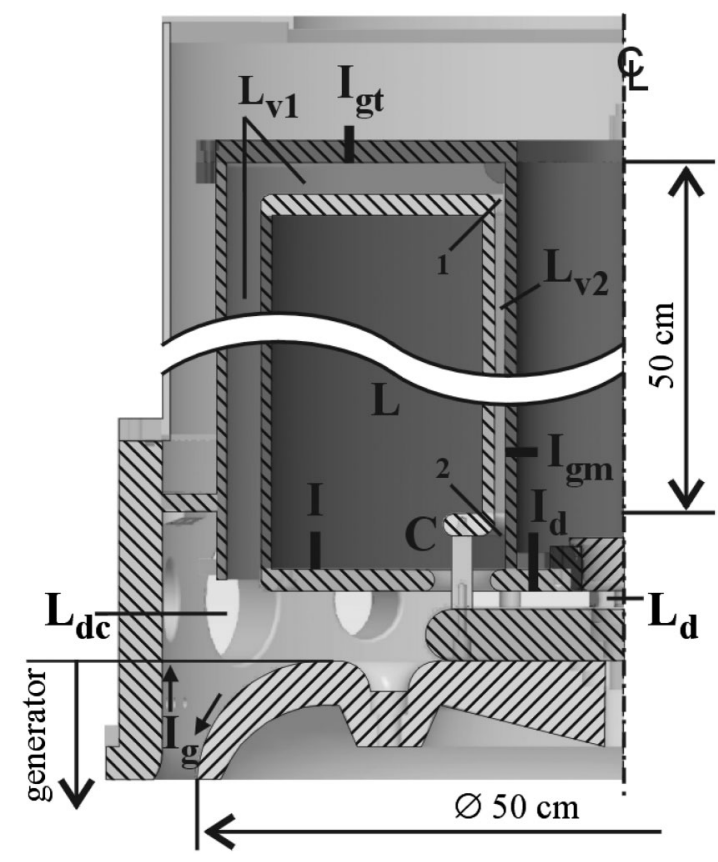

FIG. 5. Cylindrical geometry of the final LCM design for the Zebra generator. The interelectrode gaps in $L_{v 1}$ and $L_{v 2}$ parts are $2 \mathrm{~cm}$ and $6 \mathrm{~mm}$ accordingly, $L_{v}=L_{v 1}+L_{v 2}$. Shown are the locations of current measurements, each location corresponds to three azimuthally distributed differential Bdots. The generator current $I_{g}$ is measured by Bdots at the LCM top, $I_{g t}$, and near the convolute $C, I_{g m}$. The current inside the large flux extruder cavity is measured by the Bdots $I$. The load current is measured by the Bdots $I_{d}$. Distribution of current in the LCM is the same as in Fig. 3. $L_{d}$ is the load represented either a 6-mm-diameter rod or a 12-mm-width ( $\sim 1 \mathrm{~mm}$ thickness) strip installed at the axis of the system, both in stainless steel and $1 \mathrm{~cm}$ height. The inductance $L_{d}$ is calculated as that between the axial electrode and the LCM convolute posts (eight posts, each having diameter of $12 \mathrm{~mm})$.

$L_{\mathrm{dc}} \approx 9 \mathrm{nH}$ (diagnostic chamber), $L \approx 90 \mathrm{nH}$ (large LCM cavity), $L_{v}=L_{v 1}+L_{v 2} \approx 24 \mathrm{nH} \quad$ (LCM MITL's). Therefore, the region around $x \sim 2.5$ was chosen from the idealized analysis of Fig. 4. The $L_{v}$ value was then obtained from choosing some realistic interelectrode gap sizes in the bypass region. The load was either a 6-mmdiameter rod or a 12-mm-width ( $\sim 1 \mathrm{~mm}$ thickness) strip, both installed at the axis of the system. The inductance between the axial electrode and eight posts of the LCM convolute was $L_{d} \approx 7 \mathrm{nH}$. Under assumption of no current losses in the vacuum gaps, the theoretical load current multiplication coefficient $\kappa=I_{d} / I_{g}$ from Eq. (7) is $\kappa \approx 1.86$.

Figure 6 illustrates the experimental currents recorded on Zebra after the modification of Fig. 5 were made. Locations of the current measurements is are shown in Fig. 5 (three azimuthally distributed differential Bdots at each level for $I_{g t}, I_{g m}, I$, and $I_{d}$ ). Each curve of Fig. 6 is found as an average of Bdot signals for each level.

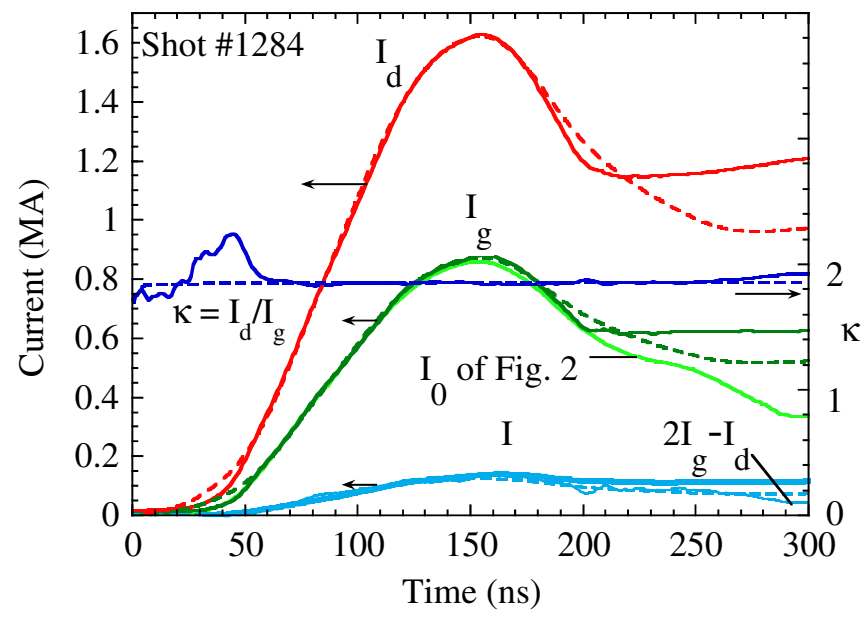

FIG. 6. (Color) Experimental currents (solid lines) measured in the configuration of Fig. 5. $I_{d}, I_{g}$, and $I$ are the load, generator, and extruder currents accordingly. The load was a 6-mmdiameter on-axis rod. Dashed lines are the numerical solution of Eqs. (7) and (8) with the $V_{\text {oc }}(t)$ from Fig. 2 increased by $6 \%$ to match this experiment. Also shown for comparison is the measured load current $I_{0}$ in nominal Zebra operation, Fig. 2.

There was no difference between two generator current measurements, $I_{g m} \approx I_{g t}=I_{g}$, which signifies no current losses in the 6-mm-gap $L_{v 2}$ MITL. The estimated electric field in this vacuum line could reach $370 \mathrm{kV} / \mathrm{cm}$ at the top (point 1 in Fig. 5) and $250 \mathrm{kV} / \mathrm{cm}$ at the bottom (point 2 in Fig. 5) in the discussed shot. The losses were also absent in other parts of the system as the experimental current multiplication $\kappa \equiv I_{d} / I_{g}$ was almost identical to the theoretical value (that assumed no losses) during a great portion of the current pulse, Fig. 6 (early spike on $\kappa$ waveform is due to the measurement uncertainty/asymmetry when the currents are still small). Numerical solutions of the system (7) and (8) for $I_{g}, I_{d}$, and $I$ are plotted as dashed lines and they coincide with corresponding experimentally recorded waveforms during the time when $V_{\mathrm{oc}}(t)$ data can be trusted, see Fig. 2. Also, the analytical extruder current $I=2 I_{g}-$ $I_{d}\left(I_{g}\right.$ and $I_{d}$ are experimental), Eq. (6), coincide with the measured $I(t)$. All above-mentioned validates the analytical description of LCM we used.

Table I summarizes results of the experiments performed in the discussed setup. Experimental peak generator currents $I_{g}$, peak load currents $I_{d}$, and load current rise times $t_{d}{ }^{\exp }$ defined in the same way as in Fig. 2 and current multiplication coefficients $\kappa$ at peak current are listed. The measured $\kappa$ is always close to the theoretical value within error bars indicating lossless operation of the setup of Fig. 5 on Zebra.

In the shot of Fig. 6, the load current reached 1.62 MA with practically unchanged current rise time $t_{d}^{\exp }=$ $t_{\text {peak }}-t_{\text {start }}=116 \mathrm{~ns}$ if compared to $t_{d}{ }^{\exp }$ in the nominal shot of Fig. 2. Indeed, the values of $L_{d}, L, L_{v}$, and $L_{\mathrm{dc}}$ realized in practice result in $L_{\mathrm{tot}}{ }^{*} \approx 85 \mathrm{nH}$, Eq. (8), and in 
TABLE I. Peak experimental currents and $\kappa$ values for configuration of Fig. 5 (relative scattering of peak value due to uncertainty/asymmetry in parentheses). $L_{d} \approx 7 \mathrm{nH}$ and theoretical $\kappa \approx 1.86$ in all shots with LCM. Shot \#1284 with LCM is shown in Fig. 6. Shot \#1286 with LCM had a strip load with $12 \mathrm{~mm}$ width instead of 6-mm-diameter rod. Shot \#1275 (Fig. 2) for Bdots calibration in standard Zebra configuration (Fig. 1) is included for comparison. $\left.L_{\text {tot }}{ }^{*}\right)$ denotes $L_{\text {tot }}$ from Eq. (3) in standard configuration and $L_{\text {tot }}{ }^{*}$ from Eq. (8) in LCM configuration.

\begin{tabular}{lcccc}
\hline \hline Shot \# & 1284 & 1285 & 1286 & 1275 \\
Configuration & LCM & LCM & LCM & Standard \\
\hline Load & rod $\varnothing 6 \mathrm{~mm}$ & rod $\varnothing 6 \mathrm{~mm}$ & strip $12 \mathrm{~mm}$ & rod $\varnothing 6 \mathrm{~mm}$ \\
$L_{\text {tot }}{ }^{*}(\mathrm{nH})$ & 85 & 85 & 85 & 66 \\
$I_{g}(\mathrm{MA})$ & $0.88( \pm 24 \%)$ & $0.83( \pm 9 \%)$ & $0.78( \pm 12 \%)$ & 0.86 \\
$I_{d}(\mathrm{MA})$ & $1.62( \pm 8 \%)$ & $1.54( \pm 11 \%)$ & $1.59( \pm 6 \%)$ & 0.86 \\
$t_{d}{ }^{\exp }(\mathrm{ns})$ & 116 & 113 & 115 & 114 \\
$\kappa$ & $1.84( \pm 25 \%)$ & $1.85( \pm 14 \%)$ & $2.04( \pm 13 \%)$ & 1 \\
\hline \hline
\end{tabular}

theoretical $t_{d} \approx 89 \mathrm{~ns}<t_{0 \mathrm{~V}} \sim 100 \mathrm{~ns}$. Therefore, the chosen LCM configuration still allows the "generatorlimited" regime on this concrete generator and therefore does not lead to $t_{d}{ }^{\exp }$ increase.

The magnetic field on the load rod surface reached the megagauss level in the experiment of Fig. 6 with the estimated value of $1.08 \mathrm{MG}$. The load magnetic energy in the volume associated with $L_{d}=7 \mathrm{nH}$ was increased from 2.6 to $9.2 \mathrm{~kJ}$ which corresponds to an improved driver-to-load energy transfer efficiency. Figure 7 shows that, for obtaining the same performance in the standard configuration of Fig. 1, the generator voltage amplitude $V_{\text {oc }}$ would have to be increased almost twice. This would lead to the stack voltage increase 2.8 times if compared to the nominal Zebra configuration, Fig. 2, and 1.8 times if

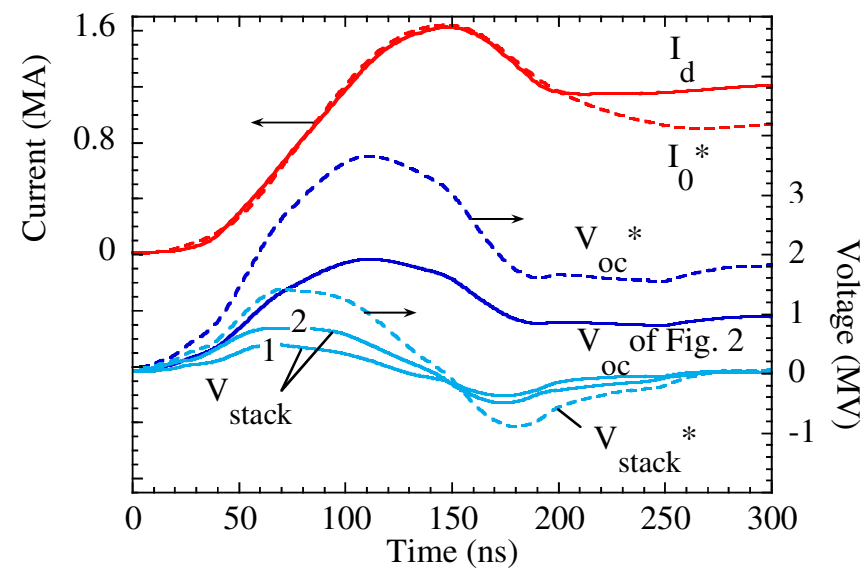

FIG. 7. (Color) Experimental currents and voltages (solid lines): nominal generator open-circuit voltage $V_{\text {oc }}$, (1)—nominal voltage on the insulator stack $V_{\text {stack }}$ in no-LCM configuration, (2)stack voltage in the LCM shot of Fig. 6 and the load current $I_{d}$ in this shot. Numerical solution of Eq. (1) (dashed lines): the same load current amplitude of 1.6 MA could be reached only if the generator energetics is increased, i.e., if the generator voltage is 1.9 times higher, $V_{\text {oc }}{ }^{*}=1.9 V_{\text {oc }}$. (3) - The peak stack voltage $V_{\text {stack }}$ would rise from nominal 0.5 to $1.4 \mathrm{MV}$ in this case. compared to the LCM operation, Fig. 6, possibly requiring an increase in $L_{0}$.

Such an increase of the generator voltage would require major changes of generator energetics and architecture. Instead, the observed enhanced load currents and load magnetic energies are obtained using the LCM technique at low cost and without modifying the generator. This capability will allow high-energy-density physics research at higher levels of magnetic field on Zebra. In particular, envisaged applications with constant-inductance loads on Zebra are the studies of plasma-magnetic field interaction and experimental modeling of space plasmas in the laboratory [11]. Applications of the LCM technique to the loads having variable inductance $L_{d}(t) \neq$ const or/and non-negligible resistance, such as planar wire-array $z$ pinches [12] are already in progress on this generator [13].

\section{ACKNOWLEDGMENTS}

We thank Dr. J.L. Porter for support of this work. We would like to thank the referee for helpful discussions. This work is supported by Sandia National Laboratories under DOE/SNL Contracts No. 681371, No. 686929, and No. 530307, by CNRS, France, by DOE/DGA-SNL/CEG agreement, by DOE under NNSA Cooperative Agreements No. DE-FC52-06NA27586, No. DE-FC52-06NA27588, and in part by No. DE-FC52-06NA27616. Sandia is a multiprogram laboratory operated by Sandia Corporation, a Lockheed Martin Company, for the United States Department of Energy's National Nuclear Security Administration under Contract No. DE-AC04-94AL85000.

[1] V. M. Titov and G. A. Shvetsov, Megagauss Fields and Pulsed Power Systems (Nova Science Publishers, New York, 1990).

[2] M. K. Matzen et al., Phys. Plasmas 12, 055503 (2005).

[3] J. P. VanDevender, in Proceedings of the 3rd International Pulsed Power Conference, edited by T.H. Martin and A. H. Guenther (IEEE, New York, 1981), pp. 248-251. 
[4] W. A. Stygar, M. E. Cuneo, D. I. Headley, H. C. Ives, R. J. Leeper, M. G. Mazarakis, C. L. Olson, J.L. Porter, T. C. Wagoner, and J.R. Woodworth, Phys. Rev. ST Accel. Beams 10, 030401 (2007).

[5] A. S. Chuvatin, L. I. Rudakov, B. V. Weber, R. Cadièrgues, and F. Bayol, Rev. Sci. Instrum. 76, 063501 (2005).

[6] A.S. Chuvatin, A. A. Kim, V.A. Kokshenev, B. M. Kovalchuk, A. V. Shishlov, A. V. Fedunin, A. Yu. Labetsky, N. A. Zhidkova, N. E. Kurmaev, F. I. Fursov, F. Lassalle, H. Calamy, and M. Krishnan, in Proceedings of the 16th IEEE International Pulsed Power Conference, 2007, Albuquerque, New Mexico (unpublished).

[7] J.S. Shlachter, Plasma Phys. Controlled Fusion 32, 1073 (1990).

[8] B. S. Bauer, V. L. Kantsyrev, N. Le Galloudec, R. Presura, G. S. Sarkisov, A. S. Shlyaptseva, S. Batie, W. Brinsmead, H. Faretto, B. LeGalloudec, A. Oxner, M. Al-Shorman, D. A. Fedin, S. Hansen, I. Paraschiv, H. Zheng, D. McCrorey, J. W. Farley, J. Glassman, and J.S. De Grootin, in Proceedings of the 12th International Pulsed
Power Conference, edited by Charles Stallings and $\mathrm{H}$. Kirbie (IEEE, New York, 1999), pp. 1045-1047.

[9] http://www.ntf.unr.edu/zebra.php.

[10] R. B. Miller, An Introduction to the Physics of Intense Charged Particle Beams (Plenum Press, New York, 1982).

[11] W. Horton, C. Chiu, T. Ditmire, P. Valanju, R. Presura, V. V. Ivanov, Y. Sentoku, V. I. Sotnikov, A. Esaulov, N. Le Galloudec, T. E. Cowan, and I. Doxas, Adv. Space Res. 39, 358 (2007).

[12] V. L. Kantsyrev, L. I. Rudakov, A. S. Safronova, A. A. Esaulov, A. S. Chuvatin, C. A. Coverdale, C. Deeney, K. M. Williamson, M.F. Yilmaz, I. Shrestha, N.D. Ouart, and G.C. Osborne, Phys. Plasmas 15, 030704 (2008).

[13] A. S. Chuvatin, V. L. Kantsyrev, L. I. Rudakov, M.E. Cuneo, A. L. Astanovitskiy, R. Presura, A. S. Safronova, A. A. Esaulov, W. Cline, K. M. Williamson, I. Shrestha, M. F. Yilmaz, G. C. Osborne, M. Weller, T. Jarrett, B. LeGalloudec, V. Nalajala, T. D. Pointon, and K.A. Mikkelson, AIP Conf. Proc. 1088, 253 (2009). 